\title{
Global Astrophysical Telescope System - telescope No. 2
}

\author{
Krzysztof Kamiński, Roman Baranowski, Monika Fagas, \\ Wojciech Borczyk, Wojciech Dimitrov and Magdalena Polińska
}

Astronomical Observatory Institute, Faculty of Physics, A. Mickiewicz University, Słoneczna 36, 60-286 Poznań, Poland

email: chrisk@amu.edu.pl

\begin{abstract}
We present the new, second spectroscopic telescope of Poznań Astronomical Observatory. The telescope allows automatic simultaneous spectroscopic and photometric observations and is scheduled to begin operation from Arizona in autumn 2013. Together with the telescope located in Borowiec, Poland, it will constitute a perfect instrument for nearly continuous spectroscopic observations of variable stars. With both instruments operational, the Global Astrophysical Telescope System will be established.
\end{abstract}

Keywords. instrumentation: spectrographs, telescopes

\section{Introduction}

Poznań Spectroscopic Telescope 2 (PST2) is the second spectroscopic telescope constructed at the Astronomical Observatory of Adam Mickiewicz University in Poznań (Poland). The first one (PST1) has been operational since 2007 in Borowiec observing station (Baranowski et al. 2009). PST2 will be transported from Poznań to Winer Observatory in Arizona (USA) in September 2013. Since both telescopes are equipped with similar echelle spectrographs, they will be perfectly suited for monitoring spectroscopic variations of pulsating stars in a nearly continuous way.

\section{The telescope}

PST2 is a slightly improved version of our first instrument. The new telescope is Planewave's model CDK700 of 0.7-m diameter with an alt-az robotic mount. The custom designed echelle spectrograph of $R=40000$ resolution is fiber fed with an optional iodine cell and a simultaneous input light measurement. The main spectroscopic camera is an Andor iKon-L, whose low readout noise and negligible dark current enables us to record spectra with $\mathrm{S} / \mathrm{N} \sim 5$ for stars up to $11 \mathrm{mag}$, which is perfectly useful for radial velocity measurements of late spectral types. The telescope is equipped with a small guider scope for simultaneous photometry during spectroscopic exposures. Everything will be controlled remotely with our dedicated software. Fully autonomous operation mode is also being prepared.

\section{Spectroscopy}

The echelle spectrograph of PST2 is designed to maintain substantial thermal stability for both short and long periods of time. Any residual changes of temperature, as well as changes in atmospheric pressure, are recorded with a precision of $0.01^{\circ} \mathrm{C}$ and $0.01 \mathrm{hPa}$. This should allow for a post-observation correction of spectrograph instabilities. Our 
preliminary tests show that it should be possible to correct radial velocities to $\sim 20 \mathrm{~m} \mathrm{~s}^{-1}$ r.m.s. using a single-point temperature and pressure measurement inside the spectrograph box. Further improvements of radial velocity measurements are possible with our optional iodine cell, but at the cost of reducing the telescope's effective limiting magnitude and contaminating the stellar spectrum with iodine lines.

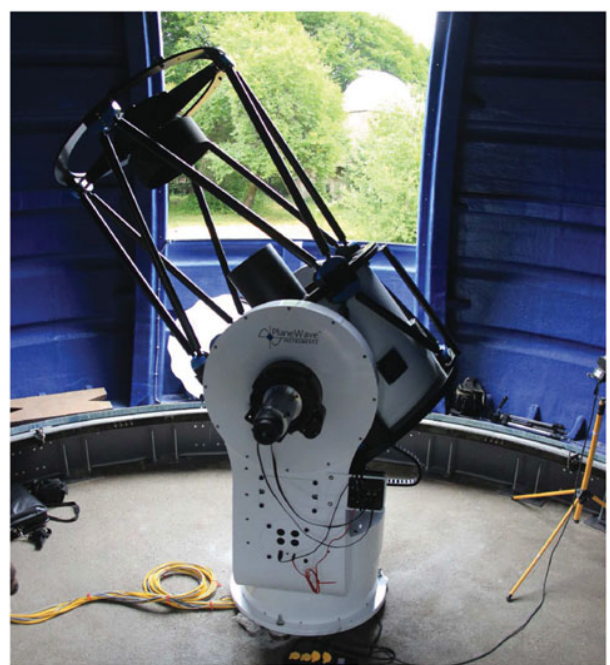

Figure 1. The PST2 telescope before installing additional equipment.

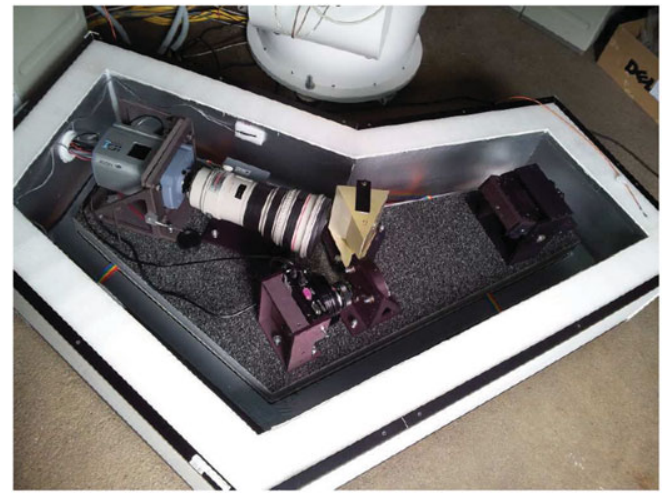

Figure 2. The PST2 echelle spectrograph inside its thermostatic box.

\section{Photometry}

The auxiliary guider scope is capable of making simultaneous photometric observations of even very bright spectroscopic targets. It is equipped with selectable two pairs of $B$ and $R$ Johnson filters. One pair of these filters is covered with a $0.1 \times 0.1 \mathrm{~mm}$ metal wire mesh. Using these, we record interferometric patterns of all stars within the guider 0.5 degree field of view. By selecting a weaker first- or second-order image of a bright target star and a zero-order image of, usually much fainter, comparison star, we are able to collect simultaneously a comparable amount of light, and therefore obtain good quality relative photometry, even when the zero-order target star image is overexposed.

Additionally PST2 is also perfectly suited for photometry of fast moving NEAs. The telescope is capable of non-sidereal tracking with selectable, constant speed. The second Nasmyth focus of our telescope is equipped with an Andor iXon $\mathrm{X}_{3}$ camera which is capable of delivering very short exposures with no dead time (by using the frame transfer technique) and no readout noise (by using electron-multiplying technology). All this combined allows us to follow fast moving asteroids for a substantial amount of time and make long series of very short exposures to combine them later, on both the target object and the comparison stars, without the cost of multiplying the camera's readout noise.

\section{Reference}

Baranowski, R., Smolec, R., Dimitrov, W., et al. 2009, MNRAS, 396, 2194 